\title{
Expectativas de laborar en el primer nivel de atención de salud de los estudiantes de una facultad de medicina de Lima, Perú
} Expectations of students from a faculty of medicine in Lima, Peru, for working in first-level health care

\author{
Carolina B. Tarqui-Mamani ${ }^{1,2, a}$, Hernán A. Sanabria Rojas ${ }^{1,2, b}$, \\ Eduardo Zárate Cárdenas ${ }^{1, c}$ \\ 1 Departamento de Medicina Preventiva y Salud Pública, Universidad Nacional Mayor de San Marcos. \\ ${ }^{2}$ Instituto Nacional de Salud, Ministerio de Salud. \\ ${ }^{a}$ Obstetra. Doctora en Salud Pública, Magister en Epidemiología; ${ }^{\text {b }}$ Médico Cirujano. Magister en Medicina; \\ ${ }^{\circ}$ Médico Cirujano, Doctor en Medicina.
}

\begin{abstract}
Resumen
Introducción: La situación sanitaria del Perú requiere que más profesionales de la salud brinden atención en los establecimientos de salud del primer nivel de atención, ubicados principalmente en las zonas periféricas, rurales o más pobres del país. Objetivo: Identificar expectativas laborales y personales de los futuros profesionales de la salud que son formados en una facultad de medicina de Lima, particularmente trabajar en el primer nivel de atención de salud. Diseño: Estudio observacional, descriptivo y exploratorio. Lugar: Facultad de Medicina, Universidad Nacional Mayor de San Marcos, Lima, Perú. Participantes: Estudiantes pre internado de las Escuelas Académico Profesionales. Intervenciones: El muestreo fue por conveniencia y se incluyó a 278 estudiantes de las 5 escuelas que conforman la facultad de medicina. Se aplicó un cuestionario ad hoc donde se preguntó aspectos relativos a las expectativas laborales, económicas y visión de los estudiantes como futuros profesionales. Se calculó promedios, desviación estándar, distribución de frecuencias, los intervalos de confianza al $95 \%$ con la prueba de Poissón y chi cuadrado. Principales medidas de resultados: Expectativa de laborar en el primer nivel de atención de salud. Resultados: El 50\% de los estudiantes manifestó que les gustaría desarrollarse en el área asistencial; solo 14,5\% expresó su intención de trabajar en el primer nivel de atención de salud (puestos y centros de salud), $87,1 \%$ consideró que estudiar en una universidad emblemática es una fortaleza que favorece su vida profesional y $57,6 \%$ que seguirá estudios de posgrado; $58,6 \%$ de los estudiantes señaló la intención de trabajar en Lima, 21,9\% en provincias y $16,2 \%$ en el extranjero. El promedio de expectativa económica mensual fue el equivalente a 1577 nuevos soles o poco más de 2 sueldos mínimos vitales previstos para el 2012. Conclusiones: La expectativa de los futuros profesionales de la salud de laborar en establecimientos de salud del primer nivel de atención es baja.
\end{abstract}

Palabras clave: Recursos humanos en salud, migración, estudiantes, atención primaria de salud, Perú.

Abstract

Introduction: Remote rural areas of the country need more health professionals. Objectives: To identify job and personal expectations of health profession students, particularly those related to work at primary care level. Design: Observational, descriptive and exploratory study. Setting: Faculty of Medicine, Universidad Nacional de San Marcos, Lima, Peru. Participants: Students of a medical school. Interventions: The convenience sample included 278 students from the 5 health professional schools. A questionnaire with questions related to job and financial expectations and on future professional perspective was applied. Main outcome measures: Intention to work at primary care level. Results: Half of students stated they would like to work in hospitals: Only $14.5 \%$ expressed their intention to work in first level facilities. Most (87.1\%) considered the university prestige as an asset for their future professional practice. More than half $(57.6 \%)$ said they would pursue post-graduate training. Almost $60 \%$ expressed an intention to work in Lima, $21.9 \%$ in provinces and $16.2 \%$ abroad. Average monthly salary expectation was equivalent to 1577 nuevos soles, slightly more than 2 minimum vital wages for 2012. Conclusions: Expectation of future health professionals for working at primary care level was low in the group studied.

Keywords: Human resources in health, migration, students, primary health care, Peru.

An Fac med. 2015;76(1):57-62 / doi:10.15381/anales.v76i1.11076 


\section{INTRODUCCIÓN}

La globalización viene afectando la vida de cada persona dada su necesidad de estar cada vez más interconectada con el mundo. La globalización es el proceso de integración global en los terrenos social, político, económico y cultural ${ }^{(1)}$. Esta profundización de las relaciones sociales y de las interacciones entre los estados y las sociedades locales o remotas no sería del todo posible sin el concurso de las modernas redes de comunicación y las tecnologías de la información ${ }^{(2)}$. El Perú no es ajeno al proceso de globalización; ha crecido económicamente en forma sostenida ${ }^{(3)}$. Existe un crecimiento vertiginoso en el comercio de exportación, industrias, construcción y desarrollo de grandes centros comerciales, viviendas y transporte; igualmente, hay un crecimiento notable en el número de universidades nacionales y particulares. Sin embargo, no se percibe un igual crecimiento y desarrollo en el área sanitaria en todos los niveles de atención. Esta situación es conocida por los futuros profesionales de la salud, quienes deben tener una amplia comprensión de diversas alternativas, culturalmente determinadas, para el ejercicio de su práctica profesional en un mundo globalizado. Por otro lado, se requiere un mejor conocimiento de las enfermedades infecciosas y tropicales, conocer los problemas del envejecimiento de la población, la carga de enfermedad y, particularmente, la estrategia de la atención primaria de la salud, entre otros asuntos. En ese sentido, las expectativas de trabajo se han incrementado al aumentar las oportunidades para que los nuevos profesionales puedan trabajar en lugares alejados dentro del país y en cualquier parte del mundo ${ }^{(4)}$.

Los alumnos universitarios tienen percepciones relativas a su futuro, como la obtención del título profesional a corto plazo, la continuación de sus estudios en segunda especialización, maestrías o diplomados ${ }^{(5)}$. Aunado a su objetivo académico, también les interesa su futuro a mediano y largo plazo, que se resume en un mejor nivel de vida. Sin embargo, al finalizar los estudios se enfrentan con un mercado laboral donde no encuentran el trabajo deseado o los salarios no cumplen sus expectativas y, por ende, su proyección de vida puede verse afectada. Los salarios que reciben los profesionales de la salud que tienen un elevado nivel educativo, en parte, provienen de privaciones, mayor trabajo y mayor especialización o nuevos estudios superiores ${ }^{(6)}$.

La situación sanitaria del Perú requiere que más profesionales de la salud brinden atención en los establecimientos de salud del primer nivel de atención, ubicados principalmente en las zonas periféricas, rurales o más pobres del país, donde se observa la mayor morbimortalidad en la población, sobretodo en la población en situación de vulnerabilidad ${ }^{(7)}$. Frente a esta problemática, el gobierno peruano propuso diversas estrategias, políticas e incentivos laborales ${ }^{(8)}$ para intentar cubrir la demanda de profesionales de la salud, principalmente médica, en el interior del país. No obstante, se observa que diversos puestos laborales ubicados en zonas de extrema pobreza no son cubiertos por los nuevos profesionales de la salud. Esta situación se atribuye a las deficientes condiciones de trabajo en las zonas rural y periférica que los inducen a laborar en la ciudad, o bien, optan por emigrar al extranjero en busca de mejores y atractivos salarios para alcanzar sus expectativas laborales y de formación profesional ${ }^{(9)}$. Algunos estudios muestran que los salarios bajos y las condiciones precarias de trabajo de los profesionales de la salud en el primer nivel de atención de salud, favorecen la emigración de los profesionales a otros países por la búsqueda de mejores estatus y nivel económico ${ }^{(10,11)}$. De otro lado, el desarrollo de la ciencia y tecnología conlleva a la necesidad de buscar especialidades y cursos de post grado ${ }^{(12)}$, que generalmente se ofrecen en las grandes ciudades o la capital del país.

La reforma del sector salud en el año 2013 incluye mejoras de la atención en el primer nivel de atención de salud, acorde con la propuesta del Ministerio de Salud (MINSA) sobre su fortalecimiento hacia el $2021^{(13)}$. Mientras tanto, los futuros profesionales de la salud tendrán como expectativa laborar en establecimientos de salud especializados u hospitales que se encuentran en Lima. Les resulta menos atractivo trabajar en establecimientos del primer nivel de atención (puestos y centros de salud). Se espera que el futuro profesional de la salud responda al llamado del MINSA como recurso humano en el primer nivel de atención de salud para fortalecer parte de la atención primaria de la salud (APS) renovada, promovida para la Organización Mundial de la Salud y sus organismos regionales ${ }^{(14)}$. La Atención Primaria de la Salud -APS. sigue siendo la principal y más efectiva estrategia para promover la salud y alcanzar el más alto nivel de salud posible para cada persona. Los recursos humanos son el componente esencial de los sistemas de salud, pero están inadecuadamente preparados para trabajar en contextos basados en APS ${ }^{(14)}$, particularmente en el Perú.

Conocer en profundidad las aspiraciones y expectativas laborales del futuro profesional en el momento en que está comenzando a pensar en su porvenir profesional y personal, permitirá orientar a las instancias correspondientes estrategias laborales y/o educativas necesarias para dar mejor respuestas a los ciudadanos, dada la alta demanda de los profesionales de la salud, en especial en las zonas rurales y pobres. Esto es importante para llevar atención apropiada y oportuna a las comunidades respectivas y así mejorar la salud de la población peruana. Asimismo, los resultados permitirán a las autoridades universitarias contar con información valiosa para dar soluciones conducentes a promover el trabajo en los establecimientos del primer nivel de salud en el Perú enmarcado dentro del proceso de APS renovada y de la globalización actual.

El estudio tuvo como objetivo identificar las expectativas laborales y personales de los futuros profesionales de 
salud que vienen siendo formados en una facultad de medicina de Lima, particularmente hacia el primer nivel de atención de salud.

\section{MÉTODOS}

Se realizó un estudio observacional y descriptivo. Se aplicó una encuesta anónima a los estudiantes en cada una de las aulas de las cinco escuelas de salud de la Facultad de Medicina Humana de una universidad pública de Lima (medicina humana 140 estudiantes, enfermería 67 , obstetricia 55 , tecnología médica 79 y nutrición 39). La población estuvo constituida por los alumnos del último año de estudios o pre internado, matriculados, que participaron voluntariamente; se excluyó a estudiantes que no cursaban el último año o no estaban matriculados. Se realizó un muestreo no probabilístico por conveniencia, aplicándose un cuestionario ad hoc donde se preguntó a los alumnos diversos aspectos relativos a las expectativas de laborar en establecimientos del primer nivel de atención (puestos y centros de salud), expectativas económicas y visión de futuro como futuros profesionales. Previo a la aplicación del cuestionario se realizó un estudio piloto en alumnos con características similares a la población de estudio para evaluar la comprensión y coherencia de las preguntas y el tiempo adecuado para su aplicación. Se realizó la digitación en Excel y luego una doble digitación para realizar el control de calidad de la digitación antes de construir una base de datos en SPSS versión 19. Se calculó promedios, desviación estándar, distribución de frecuencias, los intervalos de confianza al 95\% con las pruebas de Poissón y chi cuadrado.

La participación de los estudiantes durante la recolección de datos no involucró riesgos para su salud, fue anónima, garantizando la confidencialidad de los datos, en cumplimiento de las normas de la ética en investigación.

\section{RESULTADOS}

Se incluyó a 278 alumnos, $23,4 \%$ (65/278) de medicina humana, $19,8 \%$ (55/278) de obstetricia, $14,4 \%$ (40/278) de enfermería, 14,0\% (39/278) de nutrición y 28,4\% (79/278) de tecnología médica; el promedio de edad fue 22,6 años, con desviación estándar (DE) 2,7 años, y fluctúo entre 19 y 45 años, siendo $64 \%$ de los estudiantes de sexo femenino.

El $14,5 \%$ de los encuestados manifestó la intención de trabajar en el primer nivel de atención de salud (puestos y centros de salud), otro 50\% en el área asistencial y solo $10 \%$ en el área comunitaria. La expectativa económica de los estudiantes fue en promedio 1577 nuevos soles, siendo el rango de fluctuación entre 1000 y 8000 .

El $63,7 \%$ de los estudiantes encuestados percibió que el puesto de trabajo depende de la universidad en la cual realizó los estudios de pregrado; asimis- mo, $50 \%$ de los estudiantes consideró que su primer trabajo dependerá del lugar en donde realizaron sus prácticas pre profesionales. El 87,1\% de los estudiantes consideró que es una fortaleza que favorece su desarrollo profesional el haber estudiado en una reconocida y emblemática universidad estatal. Alrededor de 57,6\% de los estudiantes manifestó que seguirá estudios de posgrado. Finalmente, $58,6 \%$ indicó su intención de trabajar en Lima, 21,9\% en provincias, $16,2 \%$ en el extranjero, mientras que $3,5 \%$ no sabía dónde quería trabajar. En las tablas 1 y 2 se resume algunas de las características distribuidas globalmente y según la Escuela Académica Profesional de Salud de los estudiantes encuestados.

\section{DISCUSIÓN}

Los problemas complejos que se observan en cuanto al personal de salud deben ser abordados a través de políticas

Tabla 1. Área laboral, lugar y expectativa de sueldo del futuro trabajo de los estudiantes pre internado de una universidad pública de Lima, Perú. Distribución consolidada.

\begin{tabular}{lcccc} 
Característica & $N^{0}$ & $\%$ & \multicolumn{2}{c}{ IC $95 \%$} \\
Área laboral & & & & \\
Asistencial & 139 & 50,0 & 44,1 & 55,9 \\
Comunidad & 29 & 10,4 & 6,8 & 14,0 \\
Investigación & 27 & 9,7 & 6,2 & 13,2 \\
Gestión & 10 & 3,6 & 1,4 & 5,8 \\
Privado & 52 & 18,7 & 14,1 & 23,3 \\
Otro & 21 & 7,6 & 4,4 & 10,7 \\
Lugar de trabajo & & & & \\
\hline Centro y puesto de salud & 40 & 14,5 & 10,3 & 18,6 \\
Hospital & 182 & 65,9 & 60,4 & 71,5 \\
Organización no gubernamental & 31 & 11,2 & 7,5 & 15,0 \\
Otro & 23 & 8,3 & 5,1 & 11,6 \\
\hline Expectativa de sueldo & & & & \\
\hline Menos de 1000 soles & 26 & 11,0 & 7,0 & 15,0 \\
1 001 a 2000 & 66 & 28,0 & 22,2 & 33,7 \\
2 001 a 3000 & 53 & 22,5 & 17,1 & 27,8 \\
3001 a 4000 & 30 & 12,7 & 8,5 & 17,0 \\
Más de 4000 & 61 & 25,8 & 20,3 & 31,4 \\
\hline
\end{tabular}


Tabla 2. Área, lugar y expectativa de sueldo del futuro trabajo de los estudiantes pre internado de una universidad pública de Lima, Perú. Distribución según escuelas de procedencia.

\begin{tabular}{|c|c|c|c|c|c|c|c|c|c|c|}
\hline \multirow{2}{*}{ Característica } & \multicolumn{2}{|c|}{ Medicina } & \multicolumn{2}{|c|}{ Obstetricia } & \multicolumn{2}{|c|}{ Enfermería } & \multicolumn{2}{|c|}{ Nutrición } & \multicolumn{2}{|c|}{ Tecnología médica } \\
\hline & $\overline{N^{0}}$ & $\%$ & $\mathrm{~N}^{0}$ & $\%$ & $\overline{\mathrm{N}^{0}}$ & $\%$ & $\overline{N^{0}}$ & $\%$ & $\mathrm{~N}^{0}$ & $\%$ \\
\hline \multicolumn{11}{|l|}{ Lugar de procedencia } \\
\hline Lima & 46 & 70,8 & 43 & 78,2 & 33 & 82,5 & 34 & 87,2 & 58 & 74,4 \\
\hline Provincia & 19 & 29,2 & 12 & 21,8 & 7 & 17,5 & 5 & 12,8 & 20 & 25,6 \\
\hline \multicolumn{11}{|l|}{ Área laboral } \\
\hline Asistencial & 46 & 70,8 & 29 & 52,7 & 26 & 65,0 & 4 & 10,3 & 34 & 43,0 \\
\hline Comunidad & 1 & 1,5 & 11 & 20,0 & 5 & 12,5 & 6 & 15,4 & 6 & 7,6 \\
\hline Investigación & 2 & 3,1 & 9 & 16,4 & 4 & 10,0 & 4 & 10,3 & 8 & 10,1 \\
\hline Gestión & 2 & 3,1 & 0 & 0,0 & 1 & 2,5 & 5 & 12,8 & 2 & 2,5 \\
\hline Privado & 7 & 10,8 & 3 & 5,5 & 2 & 5,0 & 14 & 35,9 & 26 & 32,9 \\
\hline Otro & 7 & 10,8 & 3 & 5,5 & 2 & 5,0 & 6 & 15,4 & 3 & 3,8 \\
\hline \multicolumn{11}{|l|}{ Lugar de trabajo } \\
\hline Centro y puesto de salud & 9 & 13,8 & 21 & 38,2 & 5 & 12,5 & 1 & 2,7 & 4 & 5,1 \\
\hline Hospital & 47 & 72,3 & 18 & 32,7 & 29 & 72,5 & 18 & 48,6 & 70 & 88,6 \\
\hline ONG & 0 & 0,0 & 14 & 25,5 & 3 & 7,5 & 13 & 35,1 & 1 & 1,3 \\
\hline Otro & 9 & 13,8 & 2 & 3,6 & 3 & 7,5 & 5 & 13,5 & 4 & 5,1 \\
\hline \multicolumn{11}{|l|}{ Expectativa de sueldo } \\
\hline Menos de 600 & 0 & 0,0 & 2 & 4,1 & 1 & 3,2 & 0 & 0,0 & 0 & 0,0 \\
\hline 600 a 1000 & 1 & 1,8 & 2 & 4,1 & 8 & 25,8 & 2 & 5,9 & 10 & 15,2 \\
\hline 1001 a 2000 & 4 & 7,1 & 23 & 46,9 & 11 & 35,5 & 5 & 14,7 & 23 & 34,8 \\
\hline 2001 a 3000 & 7 & 12,5 & 11 & 22,4 & 8 & 25,8 & 11 & 32,4 & 16 & 24,2 \\
\hline 3001 a 4000 & 10 & 17,9 & 5 & 10,2 & 2 & 6,5 & 4 & 11,8 & 9 & 13,6 \\
\hline Más de 4000 & 34 & 60,7 & 6 & 12,2 & 1 & 3,2 & 12 & 35,3 & 8 & 12,1 \\
\hline \multicolumn{11}{|c|}{ Estudios en universidad emblemática } \\
\hline Sí & 58 & 90,6 & 49 & 89,1 & 34 & 87,2 & 34 & 89,5 & 67 & 90,5 \\
\hline No & 6 & 9,4 & 6 & 10,9 & 5 & 12,8 & 4 & 10,5 & 7 & 9,5 \\
\hline
\end{tabular}

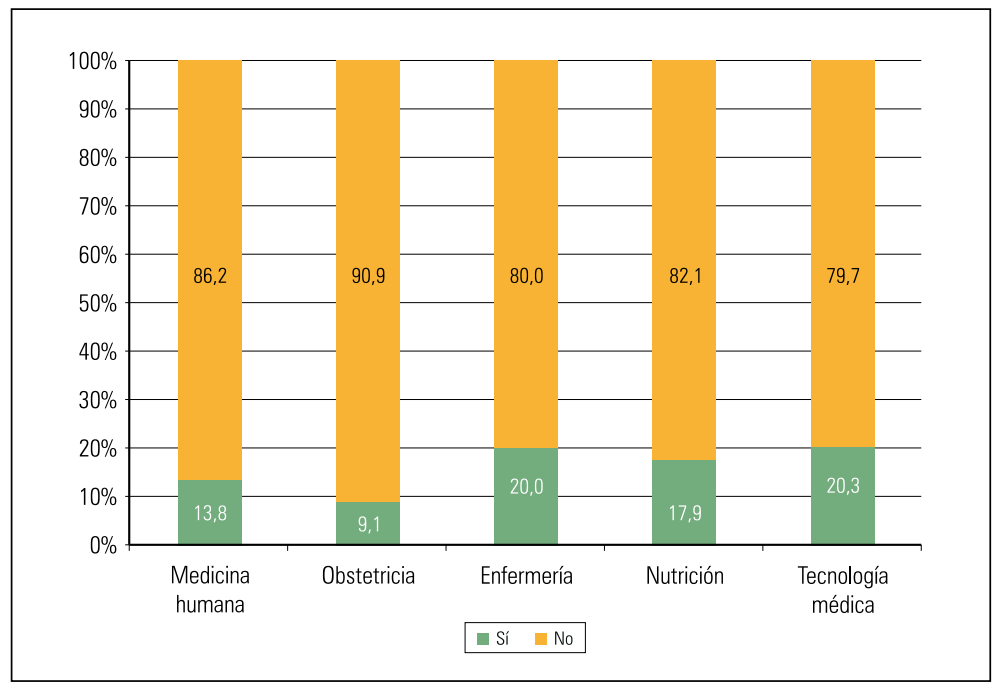

Figura 1. Distribución porcentual de la intención de emigrar al extranjero de los estudiantes de la Escuela Académico Profesionales de Salud en una universidad pública de Lima, Perú. de largo plazo, sostenibles e integrales, orientados no solamente a la superación de los tradicionales desajustes entre educación y servicios, sino también a resolver problemas de la migración, el pluriempleo/desempleo, la carrera del funcionario y del logro de la competencia laboral ${ }^{(14)}$. Esta investigación toca en parte algunos de los puntos mencionados dentro del contexto de la globalización y la APS renovada; permite conocer las expectativas de los estudiantes sobre su futuro trabajo en establecimientos del primer nivel de atención de salud, que es un punto relevante dentro de la reforma de salud actual. Durante la conferencia 'Hacia un cuidado integral de la salud para la equidad: de Alma Ata a la Declaración del Milenio', dada en la ceremonia de clausura de la Reunión de Buenos Aires 30/15, la Directora de la Organización Panamericana de la Salud -OPS- Mirtha Roses dijo "los recursos humanos son el componente esencial de los sistemas de salud, pero están inadecuadamente preparados para trabajar en contextos basados en APS"

Los resultados relativos al lugar dónde trabajar son cercanos a lo descrito por Ramírez ${ }^{(11)}$, quien refiere que solo $11,1 \%$ de los estudiantes de medicina tuvieron la intención de trabajar en puestos o centros de salud. Este hallazgo es importante, dado que significa la baja proporción de estudiantes que expresaron su intención de laborar en el área comunitaria y en los primeros niveles de atención (puestos y centros de salud), que ni siquiera llegó al 15\%. Entre las posibles explicaciones de no tener la intención de trabajar en los primeros niveles de atención y mostrar mayor interés por laborar en grandes ciudades o capitales podemos mencionar: capacitaciones, posgrado, mejores salarios y oportunidades laborales ${ }^{(10)}$. Por otra parte, el acceso a la tecnología podría explicar el bajo porcentaje de estudiantes de medicina que desean trabajar en los establecimientos de salud del primer nivel de atención, lo cual es coherente con los resultados encontrados por Perales y col ${ }^{(15)}$. Es llamativo el 
alto porcentaje de futuras obstetras que desearían trabajar en el primer nivel de atención, lo cual demostraría la sólida formación otorgada por la Escuela de Obstetricia en contenidos de APS renovada.

El gobierno peruano ha planteado diversas políticas e incentivos laborales ${ }^{(8)}$ para intentar cubrir la demanda de profesionales de la salud en los primeros niveles de atención y en el interior del país. Sin embargo, algunos puestos no son cubiertos. Igualmente, muchos profesionales de la salud se encuentran atraídos por laborar en los establecimientos de salud de mayor complejidad o especializados que se encuentran ubicados en la ciudad u optan por emigrar al extranjero en busca de alcanzar sus expectativas económicas ${ }^{(9)}$. Los resultados por escuelas profesionales no reflejan diferencias entre ellas en cuanto a trabajar en Lima y provincias.

Es preocupante que solo un quinto, aproximadamente, de los encuestados manifestara su intención de trabajar en provincias, dado que los indicadores de salud más críticos se encuentran en las zonas periféricas y rurales más pobres, según datos del Instituto $\mathrm{Na}$ cional de Estadística e Informática ${ }^{(16)}$. Entre otras razones, se encuentran la poca factibilidad de realizar estudios de posgrado y alternativas de capacitación o perfeccionamiento mediante programas presenciales o plataformas virtuales como, por ejemplo, los cursos permanentes que ofrece la OPS/OMS mediante el 'Aula virtual de salud pública' ${ }^{(17)}$. La globalización ha permitido que las tecnologías relacionadas con información y comunicación sean más accesibles para la población general, sobre todo en aquellas zonas periféricas o rurales en donde antiguamente no se contaba con computadoras ni internet por el costo elevado. Actualmente, gracias a la globalización, es posible disponer de internet en dichas zonas, que la convierte en un medio para mejorar la salud de la población y prevenir la enfermedad ${ }^{(18)}$. Sin embargo, la situación peruana es diferente, pues estar alejado de las aulas impide seguir formándose mediante programas presenciales, pero también por programas virtuales, puesto que para acceder a dichos cursos es necesario contar con el servicio de internet en forma continua y en las zonas periféricas de nuestro país el servicio de la internet es limitado y accidentado. En un mundo globalizado todos los profesionales de salud deberían tener acceso a cursos virtuales organizados por organismos internacionales vía internet para mejorar su capacidad técnica o recibir una actualización o refrescamiento del mismo ${ }^{(17)}$.

También nuestros resultados sobre emigración distan de lo encontrado por Rosales y col., quienes hallaron que 69\% de las estudiantes mexicanas del pre internado de enfermería tenía la intención de emigrar al extranjero ${ }^{(10)}$. Asimismo, Bernandini y col. ${ }^{(19)}$ hallaron que $51 \%$ de los estudiantes de medicina de España tenía la intención de emigrar al extranjero en busca de mejores oportunidades laborales. En el Perú, conforme el estudiante va culminando la carrera profesional, se incrementa la intención de emigrar al extranjero, tal y como lo informan Mayta y col., que $38,3 \%$ de los internos de medicina de la Universidad Nacional Mayor de San Marcos tuvieron la intención de emigrar al extranjero ${ }^{(20)}$. En nuestros resultados, uno de cada siete estudiantes manifestó su intención de emigrar al extranjero, situación que significa que se tiene que hacer algo para revertirlo. Si bien la migración es un derecho humano, sin embargo, cuando emigran profesionales de la salud se afecta la salud de la población por déficit de profesionales idóneos que brinden una atención adecuada. La migración de los profesionales de la salud se puede dar en dos escenarios, por un lado la emigración a otros países y, por otro lado, la emigración desde el interior del país hacia la gran ciudad como Lima y grandes capitales de regiones como Trujillo y Arequipa. Según Bernandini (19), la emigración de profesionales de la salud puede darse tanto de países muy pobres a países desarrollados como también de países con un aceptable grado de de- sarrollo hacia otros países con mayor demanda de profesionales y mejores condiciones de trabajo; en caso de los profesionales de la salud, la emigración estaría relacionada al desarrollo del sistema de salud y social del país.

En cuanto a las expectativas salariales, se sabe que los salarios bajos y las condiciones precarias de trabajo favorecen la emigración de los profesionales de la salud a otros países en búsqueda de un mejor estatus social y nivel económico ${ }^{(10,11)}$. Es comprensible que los salarios bajos no respondan a las expectativas económicas de los futuros profesionales de la salud, así como la limitada oferta de plazas y poca satisfacción en el ambiente laboral, entre otras razones, contribuyen a que al futuro profesional no le interese laborar en establecimientos del primer nivel de atención sino mas bien en establecimientos de mayor nivel de complejidad, privilegiando las grandes ciudades o emigración fuera del país. La mejor expectativa salarial se aprecia en los futuros médicos de la Escuela de Medicina, ya que la mayoría espera ganar más de 4000 nuevos soles al mes; esto último posiblemente en correspondencia a un mayor número de años de estudio. Mayta y Dulanto ${ }^{(20)}$ encontraron que la gran mayoría de estudiantes de medicina tenían expectativas de ganar 6000 de nuevos soles, lo que de alguna se corresponde con ganar más de 4000 nuevos soles encontrado en nuestro estudio. Es notable la diferencia de la expectativa de ganar en estudiantes de la Escuela de Medicina en comparación de las otras escuelas.

Una limitación del estudio fue la no presencia de algunos estudiantes de pre internado y otros que se negaron a llenar el cuestionario. La limitación queda compensada al haber encuestado a la mayoría de los estudiantes de todas las escuelas de la Facultad, es decir, Medicina Humana, Enfermería, Obstetricia, Nutrición y Tecnología Médica.

Una pregunta a las escuelas universitarias es ¿Cómo combatir este virus de la inviabilidad y dualidad que hace 
que los ministerios sigan impulsando la atención en el primer nivel de atención y la universidad tenga cursos implementados para este fin (Salud Comunitaria, Medicina Integral, etc.)?, problema que se estaría arrastrando desde hace más de una década. Una manera de abordarlo sería que las universidades peruanas que forman profesionales de la salud implementen los currículos orientados a la atención primaria de salud concordante con la propuesta de la OPS/OMS ${ }^{(21)}$ y la reforma del sector salud por parte del MINSA, que incluye el fortalecimiento tecnológico (ecografías) en el primer nivel de atención ${ }^{(13)}$. Y que el inicio de la carrera profesional incluya necesariamente un comienzo en ese nivel de atención para lograr insertar a los estudiantes y que las brechas de cobertura en las sociedades rurales se acorten con equidad. La agenda pendiente es comenzar a discutir los esquemas, la imagen deseable o la utopía de acercar las imágenes de la globalización (tecnología) y las aspiraciones de los profesionales con las propuestas de implementación de programas de atención de salud en el primer nivel a lo largo de las zonas más pobres del país. Así se podrá sensibilizar y fomentar el trabajo de los futuros profesionales de la salud hacia el primer nivel de atención de salud.

Es de esperar que los resultados de la presente investigación puedan ser de utilidad para los funcionarios del Estado, de modo que puedan contemplar las expectativas profesionales que se generan desde la universidad para que mejoren sus políticas y así se disminuya la fuga del profesional de salud formado en el Perú, particularmente de universidades nacionales. De igual modo, los estamentos del sector salud deben cumplir con la normatividad vigente de condiciones de trabajo ${ }^{(22)}$ para los trabajadores de la salud, particularmente de las zonas rurales y urbanas marginales más alejadas y más pobres.

Se concluye que la expectativa de los futuros profesionales de la salud de laborar en establecimientos de salud del primer nivel de atención es baja.

\section{RECOMENDACIONES}

La universidad debería establecer e implementar nuevos currículos universitarios que formen futuros profesionales de la salud competentes en APS, gestionando y negociando con los establecimientos de salud en el primer nivel de atención de salud a fin de tener las condiciones para la práctica de la APS.

Los estamentos del sector salud debieran mejorar el cumplimiento de la normatividad vigente sobre condiciones de trabajo para los trabajadores de la salud en los establecimientos, particularmente de las zonas rurales y urbanas marginales.

\section{REFERENCIAS BIBLIOGRÁFICAS}

1. Casals C. Globalización. Apuntes de un proceso que está transformando nuestras vidas [monografía en internet]. Barcelona: Intermón-Oxfam; 2001 [Citado 03 Mar 2013]. Disponible en: http://www. aloj.us.es/vmanzano/docencia/movsoc/resumen/ casals.pdf

2. Rosenau J. The Dynamics of Globalization: Toward an Operational Formulation. Security Dialogue. 1996;27(3):247-62.

3. Ministerio de Economía y Finanzas. Crecimiento económico con bienestar social. [Monografia en internet]. Lima: Ministerio de Economía y Finanzas; 2010 [Citado 03 Mar 2013]. Disponible en: http://www.mef.gob.pe/contenidos/prensa/ boletines/noticias_mef/bk9_18072011/externo/ MEF09externo.pdf?

4. Drain PK, Primack A, Hunt DD, Fawzi WW, Holmes KK, Gardner P. Global health in medical education: a call for more training and opportunities. Acad Med. 2007 Mar;82(3):226-30

5. Aisenson D, Virgili N, Rivarola R, Rivero L, Polastri G, Siniuk D, Scharwcz J. Seguimiento de los estudiantes que participaron en un programa de orientación vocacional en grupo. [Monografia en internet]. Buenos Aires: Universidad de Buenos Aires; 2010 [Citado el 03 Mar 2013]. Disponible en: http://www. scielo.org.ar/pdf/anuinv/v17/v17a10.pdf

6. Torres S. Entre el estudio y el trabajo: la situación y las búsquedas de los estudiantes. Revista de la Educación Superior. Jul-sep 2005;135:155-61.

7. Ministerio de Salud, Dirección General de Epidemiologia. Análisis y Situación de Salud. Enero-diciembre 2012. Bol Epidemiol (Lima). 2012;21(52):877-906-

8. Ministerio de Salud, Instituto de Desarrollo de Recursos Humanos. El Perú respondiendo a los desafíos de la Década de los Recursos Humanos en Salud. Observatorio Nacional de Recursos Humanos en Salud (Perú): MINSA; 2006.

9. Malvárez S, Castrillón M. Overview of the nursing workforce in Latin America. Serie Human Resources Development [monografía en internet]. Washington: Pan American Health Organization, 2005 [consultado 2006 abril 25]. Disponible en: http:// www.icn.ch/global//ssue6LatinAmerica.pdf
10. Rosales-Martínez Y, Nigenda G, Galárraga O, RuizLarios JA. Expectativas de migración internacional en estudiantes de enfermería en México, Distrito Federal. Salud Publica Mex. 2010;52:244-53.

11. Ramírez M. Expectativas profesionales de internos de medicina y su inclinación por la atención primaria de salud. An Fac med. 2008;69(3):176-81.

12. Fraga $C$, Rosa A. Formacao e treinamiento do medico general. Educación medica y salud. 1979;13(2):149-63.

13. Ministerio de Salud. Plan nacional de fortalecimiento del primer nivel de atención 2011-2021. Dirección General de Salud de las Personas (Perú): MINSA; 2011.

14. OPS/OMS. La formación en Medicina orientada hacia la Atención Primaria. Serie La Renovación de la Atención Primaria de Salud en las Américas. №2. Washington. 2008.

15. Perales A, Sánchez E, Mendoza A. Vocación medica en estudiantes de medicina de una universidad peruana. An Fac med. 2014:75(1):37-42.

16. Instituto Nacional de Estadistica e Informática (INEI). Encuesta Demográfica y de Salud Familiar 2011. Lima: INEl; 2012.

17. Auer A, Guerrero Espinel JE. La Organización Panamericana de la Salud y la salud internacional: una historia de formación, conceptualización y desarrollo colectivo. Rev Panam Salud Publica. 2011;30(2):111-21.

18. Galimany J, Girbau R. Globalización y salud. RISAI 2009;1(4):1-5.

19. Bernardini-Zambrini D, Barengo N, Bardach A, Hannae M, Juan M. ¿Migrar o no migrar? ¿Qué pasará con nuestra próxima generación de médicos? Estudio sobre causas y motivos en estudiantes avanzados de medicina en 11 universidades de España. Aten Primaria. 2012;44:238-9.

20. Mayta P, Dulanto A. Prevalencia y factores asociados con la intención de Emigración en internos de medicina de una universidad pública, Lima 2007. Rev peru med exp salud publica. 2008;25(3):274-8.

21. Nebot Adell C, Rosales Echevarria C, Borrell Bentz RM. Curso virtual para el desarrollo de competencias en atención primaria de salud. Rev Panam Salud Publica. 2009;26(2):176-83.

22. Ministerio de Trabajo (2012). Norma de Seguridad y Salud en el Trabajo de la Ley 29783. Reglamento Nº 005-2012 TR. Lima.

Articulo recibido el 10 de junio de 2014 y aceptado para publicación el 31 de agosto de 2014.

Conflictos de interés: Los autores declaran que no existen conflictos de intereses.

\section{Correspondencia:}

Carolina Tarqui-Mamani.

Departamento de Medicina Preventiva y Salud Pública.

Av. Grau 755, Lima 1. Perú.

Correo electrónico: ctarquim@unmsm.edu.pe o carobtm@gmail.com 